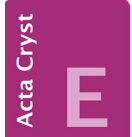
COMMUNICATIONS

ISSN 2056-9890

\section{Crystal structure of 2-cyclohexyl-1,3- thiazolo[4,5-b]pyridine}

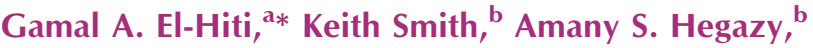 \\ Mansour D. Ajarim ${ }^{\mathrm{c}}$ and Benson M. Kariuki ${ }^{\mathrm{b} *}$ \\ ${ }^{a}$ Cornea Research Chair, Department of Optometry, College of Applied Medical \\ Sciences, King Saud University, PO Box 10219, Riyadh 11433, Saudi Arabia, \\ ${ }^{\mathbf{b}}$ School of Chemistry, Cardiff University, Main Building, Park Place, Cardiff \\ CF10 3AT, Wales, and ${ }^{\mathrm{C} C r i m i n a l}$ Evidence, Ministry of Interior, Riyadh 11632 \\ PO Box 86985, Saudi Arabia. *Correspondence e-mail: gelhiti@ksu.edu.sa, \\ kariukib@cardiff.ac.uk
}

Received 9 October 2015; accepted 10 October 2015

Edited by W. T. A. Harrison, University of Aberdeen, Scotland

In the title compound, $\mathrm{C}_{12} \mathrm{H}_{14} \mathrm{~N}_{2} \mathrm{~S}$, the cyclohexane ring adopts a chair conformation with the exocyclic $\mathrm{C}-\mathrm{C}$ bond in an equatorial orientation. The mean plane through the cyclohexane ring (all atoms) is twisted from the thiazolopyridine ring system (r.m.s. deviation $=0.013 \AA$ ) by $39.57(6)^{\circ}$. In the crystal, molecules form (100) sheets, although there are no specific directional interactions between them. The crystal stucture was refined as a two-component perfect twin.

Keywords: crystal structure; cyclohexane; thiazolopyridine derivatives; thiazolo[4,5-b]pyridine.

CCDC reference: 1430576

\section{Related literature}

For background to the uses of thiazolopyridine derivatives, see: Leysen et al. (1984). For a related structure reported by us and further references, see: El-Hiti et al. (2015). For the first report of this compound and spectroscopic data, see: Smith $e t$ al. (1995).

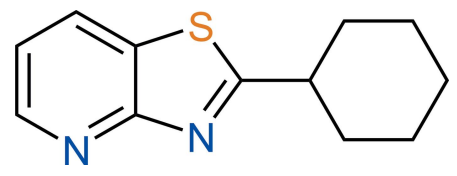

\section{Experimental}

2.1. Crystal data

$\mathrm{C}_{12} \mathrm{H}_{14} \mathrm{~N}_{2} \mathrm{~S}$

$M_{r}=218.31$

Monoclinic, $P 2_{1} / n$

$a=7.8884(5) \mathrm{A}$

$b=11.8079(7) \AA$

$c=12.2134(6) \AA$

$\beta=100.589(6)^{\circ}$

$$
\begin{aligned}
& V=1118.25(11) \AA^{3} \\
& Z=4 \\
& \mathrm{Cu} K \alpha \text { radiation } \\
& \mu=2.29 \mathrm{~mm}^{-1} \\
& T=293 \mathrm{~K} \\
& 0.27 \times 0.17 \times 0.14 \mathrm{~mm}
\end{aligned}
$$

\subsection{Data collection}

Agilent SuperNova Dual Source diffractometer with an Atlas detector

Absorption correction: multi-scan (CrysAlis PRO; Agilent, 2014)

$T_{\min }=0.592, T_{\max }=1.000$

7328 measured reflections 3913 independent reflections 3429 reflections with $I>2 \sigma(I)$ $R_{\text {int }}=0.015$

\subsection{Refinement}

$R\left[F^{2}>2 \sigma\left(F^{2}\right)\right]=0.063$

$w R\left(F^{2}\right)=0.180$

$S=1.03$

3913 reflections

Data collection: CrysAlis PRO (Agilent, 2014); cell refinement: CrysAlis PRO; data reduction: CrysAlis PRO; program(s) used to solve structure: SHELXS97 (Sheldrick, 2008); program(s) used to refine structure: SHELXL2013 (Sheldrick, 2015); molecular graphics: ORTEP-3 for Windows (Farrugia, 2012); software used to prepare material for publication: Win GX (Farrugia, 2012) and CHEMDRAW Ultra (Cambridge Soft, 2001).

\title{
Acknowledgements
}

The authors extend their appreciation to the Criminal Evidence Department, Ministry of Interior, Riyadh, Saudi Arabia, for funding this research and to Cardiff University for continued support.

Supporting information for this paper is available from the IUCr electronic archives (Reference: HB7518).

\section{References}

Agilent (2014). CrysAlis PRO. Agilent Technologies, Yarnton, England. Cambridge Soft (2001). CHEMDRAW Ultra. Cambridge Soft Corporation, Cambridge, Massachusetts, USA.

El-Hiti, G. A., Smith, K., Hegazy, A. S., Alanazi, S. A. \& Kariuki, B. M. (2015). Acta Cryst. E71, o272-o273.

Farrugia, L. J. (2012). J. Appl. Cryst. 45, 849-854.

Leysen, D. C., Haemers, A. \& Bollaert, W. (1984). J. Heterocycl. Chem. 21, 1361-1366.

Sheldrick, G. M. (2008). Acta Cryst. A64, 112-122.

Sheldrick, G. M. (2015). Acta Cryst. C71, 3-8.

Smith, K., Anderson, D. \& Matthews, I. (1995). Sulfur Lett. 18, 79-95. 


\title{
supporting information
}

Acta Cryst. (2015). E71, o866 [https://doi.org/10.1107/S2056989015019106]

\section{Crystal structure of 2-cyclohexyl-1,3-thiazolo[4,5-b] pyridine}

\author{
Gamal A. El-Hiti, Keith Smith, Amany S. Hegazy, Mansour D. Ajarim and Benson M. Kariuki
}

\section{S1. Chemical context}

Thiazolopyridine derivatives have been reported to exhibit interesting biological activities (Leysen et al., 1984). As part of our ongoing studies in this area (El-Hiti et al., 2015), we now report the structure of the title compound.

\section{S2. Structural commentary}

The asymmetric unit comprises one molecule of $\mathrm{C}_{12} \mathrm{H}_{14} \mathrm{~N}_{2} \mathrm{~S}$ (Fig. 1). The cyclohexane ring is in the chair conformation in the molecule. The least squares plane through the cyclohexane ring is twisted from the thiazolopyridine group by $39.57(6)^{\circ}$. In the crystal structure, the molecular axes are aligned along [001] (Fig. 2).

\section{S3. Synthesis and crystallization}

2-Cyclohexyl-1,3-thiazolo[4,5-b]pyridine was obtained in quantitative yield from acid hydrolysis (HCl, $5 \mathrm{M})$ of 3-(diisopropylaminothiocarbonylthio)-2-(cyclohexylcarbonylamino)pyridine under reflux for $5 \mathrm{~h}$ (Smith et al., 1995). The compound may also be synthesized in 94\% yield from acid hydrolysis (5 M HCl, $5 \mathrm{~h}$ reflux) of 3-(diisopropylaminothiocarbonylthio)-2-(bis(cyclohexylcarbony)lamino) pyridine (Smith et al., 1995). Crystallization of the crude product from diethyl ether gave the title compound as colourless crystals. Spectroscopic and analytical data are consistent with those reported (Smith et al., 1995).

\section{S4. Refinement details}

The data were twinned and HKLF5 in Shelxl 2013 was used. H atoms were positioned geometrically and refined using a riding model with $U_{\text {iso }}(\mathrm{H})$ constrained to be 1.2 times $U_{\text {eq }}$ for the atom it is bonded to.

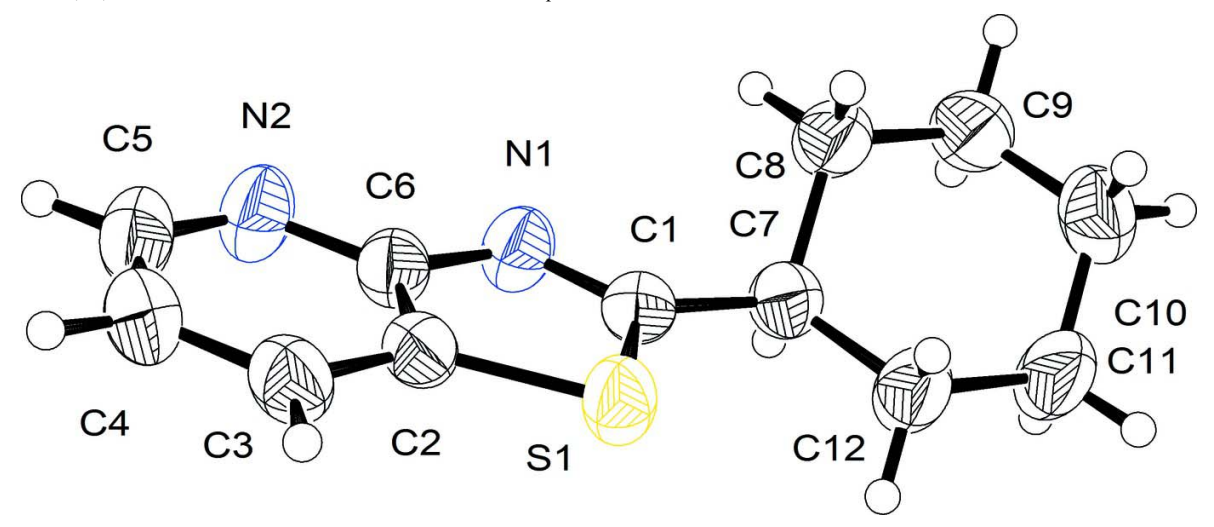

Figure 1

The asymmetric unit of $\mathrm{C}_{12} \mathrm{H}_{14} \mathrm{~N}_{2} \mathrm{~S}$ with $50 \%$ probability displacement ellipsoids for nonhydrogen atoms. 


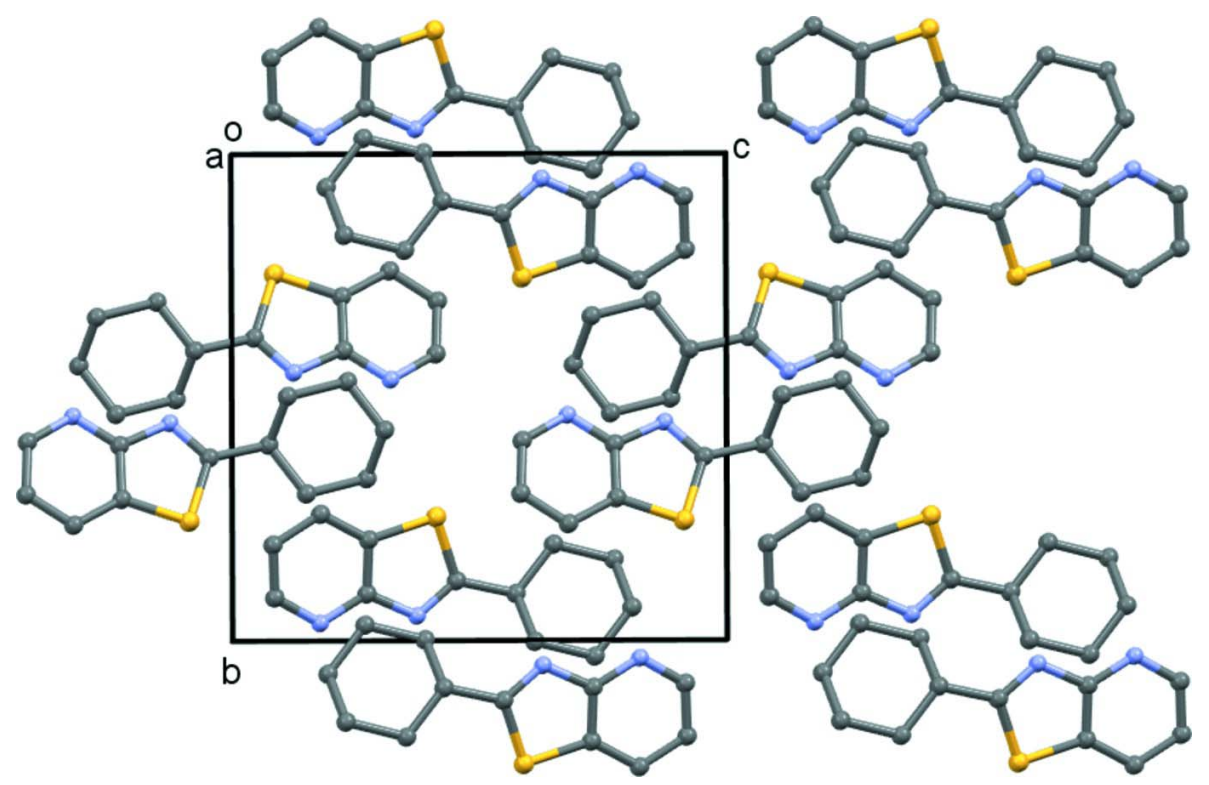

Figure 2

Crystal packing viewed along the $a$ axis.

2-Cyclohexyl-1,3-thiazolo[4,5-b] pyridine

Crystal data

$\mathrm{C}_{12} \mathrm{H}_{14} \mathrm{~N}_{2} \mathrm{~S}$

$M_{r}=218.31$

Monoclinic, $P 2{ }_{1} / n$

$a=7.8884(5) \AA$

$b=11.8079(7) \AA$

$c=12.2134(6) \AA$

$\beta=100.589(6)^{\circ}$

$V=1118.25(11) \AA^{3}$

$Z=4$

\section{Data collection}

Agilent SuperNova Dual Source

diffractometer with an Atlas detector

$\omega$ scans

Absorption correction: multi-scan

(CrysAlis PRO; Agilent, 2014)

$T_{\min }=0.592, T_{\max }=1.000$

7328 measured reflections

\section{Refinement}

Refinement on $F^{2}$

Least-squares matrix: full

$R\left[F^{2}>2 \sigma\left(F^{2}\right)\right]=0.063$

$w R\left(F^{2}\right)=0.180$

$S=1.03$

3913 reflections

136 parameters

0 restraints
$F(000)=464$

$D_{\mathrm{x}}=1.297 \mathrm{Mg} \mathrm{m}^{-3}$

$\mathrm{Cu} K \alpha$ radiation, $\lambda=1.54184 \AA$

Cell parameters from 1721 reflections

$\theta=6.2-73.9^{\circ}$

$\mu=2.29 \mathrm{~mm}^{-1}$

$T=293 \mathrm{~K}$

Needle, colourless

$0.27 \times 0.17 \times 0.14 \mathrm{~mm}$

3913 independent reflections

3429 reflections with $I>2 \sigma(I)$

$R_{\text {int }}=0.015$

$\theta_{\max }=74.2^{\circ}, \theta_{\min }=5.3^{\circ}$

$h=-9 \rightarrow 9$

$k=-14 \rightarrow 14$

$l=-15 \rightarrow 15$

Hydrogen site location: inferred from neighbouring sites

$\mathrm{H}$-atom parameters constrained

$w=1 /\left[\sigma^{2}\left(F_{\mathrm{o}}{ }^{2}\right)+(0.1345 P)^{2}+0.113 P\right]$

where $P=\left(F_{\mathrm{o}}{ }^{2}+2 F_{\mathrm{c}}{ }^{2}\right) / 3$

$(\Delta / \sigma)_{\max }=0.001$

$\Delta \rho_{\max }=0.30 \mathrm{e} \AA^{-3}$

$\Delta \rho_{\min }=-0.29$ e $\AA^{-3}$ 


\section{Special details}

Experimental. Absorption correction: CrysAlisPro, Agilent Technologies, Version 1.171.37.33 (release 27-03-2014 CrysAlis171 .NET) (compiled Mar 27 2014,17:12:48) Empirical absorption correction using spherical harmonics, implemented in SCALE3 ABSPACK scaling algorithm. Empirical absorption correction using spherical harmonics, implemented in SCALE3 ABSPACK scaling algorithm.

Geometry. All e.s.d.'s (except the e.s.d. in the dihedral angle between two 1.s. planes) are estimated using the full covariance matrix. The cell e.s.d.'s are taken into account individually in the estimation of e.s.d.'s in distances, angles and torsion angles; correlations between e.s.d.'s in cell parameters are only used when they are defined by crystal symmetry. An approximate (isotropic) treatment of cell e.s.d.'s is used for estimating e.s.d.'s involving l.s. planes.

Refinement. Refined as a 2-component perfect twin.

Fractional atomic coordinates and isotropic or equivalent isotropic displacement parameters $\left(\hat{A}^{2}\right)$

\begin{tabular}{|c|c|c|c|c|}
\hline & $x$ & $y$ & $z$ & $U_{\text {iso }} * / U_{\text {eq }}$ \\
\hline $\mathrm{C} 1$ & $0.2814(3)$ & 0.12366 (19) & $0.54649(18)$ & $0.0467(5)$ \\
\hline $\mathrm{C} 2$ & $0.2459(3)$ & 0.20807 (19) & $0.72356(18)$ & $0.0467(5)$ \\
\hline $\mathrm{C} 3$ & $0.2223(4)$ & $0.2609(2)$ & $0.8209(2)$ & $0.0561(6)$ \\
\hline $\mathrm{H} 3$ & 0.1818 & 0.3349 & 0.8212 & $0.067^{*}$ \\
\hline $\mathrm{C} 4$ & $0.2619(4)$ & $0.1982(3)$ & $0.9169(2)$ & $0.0634(7)$ \\
\hline $\mathrm{H} 4$ & 0.2461 & 0.2291 & 0.9844 & $0.076^{*}$ \\
\hline $\mathrm{C} 5$ & $0.3249(4)$ & $0.0897(3)$ & $0.9136(2)$ & $0.0659(7)$ \\
\hline H5 & 0.3505 & 0.0501 & 0.9805 & $0.079 *$ \\
\hline C6 & $0.3115(3)$ & $0.0972(2)$ & $0.72741(18)$ & $0.0472(5)$ \\
\hline $\mathrm{C} 7$ & $0.2823(3)$ & $0.0976(2)$ & $0.42621(18)$ & $0.0501(5)$ \\
\hline $\mathrm{H} 7$ & 0.3994 & 0.0741 & 0.4202 & $0.060^{*}$ \\
\hline $\mathrm{C} 8$ & $0.1612(4)$ & $-0.0018(2)$ & $0.3883(2)$ & $0.0616(7)$ \\
\hline H8A & 0.1972 & -0.0671 & 0.4350 & $0.074^{*}$ \\
\hline H8B & 0.0450 & 0.0181 & 0.3967 & $0.074^{*}$ \\
\hline C9 & $0.1620(4)$ & $-0.0321(2)$ & $0.2671(2)$ & $0.0654(7)$ \\
\hline H9A & 0.2758 & -0.0588 & 0.2600 & $0.079^{*}$ \\
\hline H9B & 0.0805 & -0.0929 & 0.2443 & $0.079^{*}$ \\
\hline $\mathrm{C} 10$ & 0.1149 (4) & $0.0680(3)$ & $0.1922(2)$ & $0.0706(8)$ \\
\hline $\mathrm{H} 10 \mathrm{~A}$ & -0.0030 & 0.0901 & 0.1937 & $0.085^{*}$ \\
\hline H10B & 0.1218 & 0.0471 & 0.1163 & $0.085^{*}$ \\
\hline C11 & $0.2341(5)$ & $0.1671(3)$ & $0.2281(2)$ & $0.0856(11)$ \\
\hline H11A & 0.1964 & 0.2320 & 0.1811 & $0.103^{*}$ \\
\hline H11B & 0.3501 & 0.1478 & 0.2188 & $0.103 *$ \\
\hline $\mathrm{C} 12$ & $0.2352(5)$ & $0.1982(2)$ & $0.3501(2)$ & $0.0709(8)$ \\
\hline $\mathrm{H} 12 \mathrm{~A}$ & 0.3176 & 0.2587 & 0.3723 & $0.085^{*}$ \\
\hline H12B & 0.1220 & 0.2257 & 0.3577 & $0.085^{*}$ \\
\hline N1 & $0.3309(3)$ & $0.05196(17)$ & $0.62604(16)$ & $0.0529(5)$ \\
\hline N2 & $0.3519(3)$ & $0.0371(2)$ & $0.82184(17)$ & $0.0616(6)$ \\
\hline $\mathrm{S} 1$ & $0.20709(8)$ & $0.25440(5)$ & $0.58736(5)$ & $0.0545(3)$ \\
\hline
\end{tabular}

Atomic displacement parameters $\left(\AA^{2}\right)$

\begin{tabular}{lllllll}
\hline & $U^{11}$ & $U^{22}$ & $U^{33}$ & $U^{12}$ & $U^{13}$ & $U^{23}$ \\
\hline $\mathrm{C} 1$ & $0.0509(11)$ & $0.0426(11)$ & $0.0457(11)$ & $-0.0033(9)$ & $0.0065(9)$ & $-0.0001(8)$ \\
$\mathrm{C} 2$ & $0.0516(11)$ & $0.0423(11)$ & $0.0436(11)$ & $-0.0036(9)$ & $0.0021(8)$ & $-0.0008(9)$
\end{tabular}




\begin{tabular}{lllllll} 
C3 & $0.0664(15)$ & $0.0505(13)$ & $0.0496(13)$ & $-0.0023(11)$ & $0.0058(11)$ & $-0.0090(10)$ \\
C4 & $0.0767(17)$ & $0.0704(18)$ & $0.0419(12)$ & $-0.0073(13)$ & $0.0076(11)$ & $-0.0079(11)$ \\
C5 & $0.0794(17)$ & $0.0733(18)$ & $0.0425(13)$ & $0.0014(13)$ & $0.0047(11)$ & $0.0092(11)$ \\
C6 & $0.0507(12)$ & $0.0475(12)$ & $0.0425(11)$ & $0.0013(9)$ & $0.0060(8)$ & $0.0012(9)$ \\
C7 & $0.0547(13)$ & $0.0510(13)$ & $0.0453(11)$ & $-0.0017(9)$ & $0.0107(9)$ & $-0.0023(9)$ \\
C8 & $0.0855(18)$ & $0.0504(13)$ & $0.0502(13)$ & $-0.0138(13)$ & $0.0160(12)$ & $-0.0044(10)$ \\
C9 & $0.0846(18)$ & $0.0552(15)$ & $0.0563(15)$ & $-0.0118(13)$ & $0.0121(12)$ & $-0.0137(11)$ \\
C10 & $0.0842(19)$ & $0.0764(19)$ & $0.0473(13)$ & $0.0011(15)$ & $0.0020(12)$ & $-0.0089(13)$ \\
C11 & $0.145(3)$ & $0.0712(19)$ & $0.0416(13)$ & $-0.030(2)$ & $0.0189(15)$ & $0.0008(12)$ \\
C12 & $0.116(2)$ & $0.0519(15)$ & $0.0442(13)$ & $-0.0148(15)$ & $0.0133(13)$ & $-0.0002(11)$ \\
N1 & $0.0672(12)$ & $0.0472(10)$ & $0.0450(10)$ & $0.0074(9)$ & $0.0121(8)$ & $0.0036(8)$ \\
N2 & $0.0795(14)$ & $0.0581(13)$ & $0.0467(11)$ & $0.0120(11)$ & $0.0104(9)$ & $0.0103(9)$ \\
S1 & $0.0753(5)$ & $0.0424(4)$ & $0.0425(4)$ & $0.0053(2)$ & $0.0017(3)$ & $0.0013(2)$ \\
\hline
\end{tabular}

Geometric parameters $\left(\AA,{ }^{\circ}\right)$

\begin{tabular}{|c|c|c|c|}
\hline $\mathrm{C} 1-\mathrm{N} 1$ & $1.294(3)$ & $\mathrm{C} 7-\mathrm{H} 7$ & 0.9800 \\
\hline $\mathrm{C} 1-\mathrm{C} 7$ & $1.502(3)$ & $\mathrm{C} 8-\mathrm{C} 9$ & $1.524(4)$ \\
\hline $\mathrm{C} 1-\mathrm{S} 1$ & $1.756(2)$ & $\mathrm{C} 8-\mathrm{H} 8 \mathrm{~A}$ & 0.9700 \\
\hline $\mathrm{C} 2-\mathrm{C} 3$ & $1.385(3)$ & $\mathrm{C} 8-\mathrm{H} 8 \mathrm{~B}$ & 0.9700 \\
\hline $\mathrm{C} 2-\mathrm{C} 6$ & $1.405(3)$ & $\mathrm{C} 9-\mathrm{C} 10$ & $1.499(4)$ \\
\hline $\mathrm{C} 2-\mathrm{S} 1$ & $1.724(2)$ & C9-H9A & 0.9700 \\
\hline $\mathrm{C} 3-\mathrm{C} 4$ & $1.374(4)$ & C9-H9B & 0.9700 \\
\hline $\mathrm{C} 3-\mathrm{H} 3$ & 0.9300 & $\mathrm{C} 10-\mathrm{C} 11$ & $1.515(4)$ \\
\hline $\mathrm{C} 4-\mathrm{C} 5$ & $1.378(4)$ & $\mathrm{C} 10-\mathrm{H} 10 \mathrm{~A}$ & 0.9700 \\
\hline $\mathrm{C} 4-\mathrm{H} 4$ & 0.9300 & $\mathrm{C} 10-\mathrm{H} 10 \mathrm{~B}$ & 0.9700 \\
\hline $\mathrm{C} 5-\mathrm{N} 2$ & $1.332(3)$ & $\mathrm{C} 11-\mathrm{C} 12$ & $1.534(4)$ \\
\hline $\mathrm{C} 5-\mathrm{H} 5$ & 0.9300 & $\mathrm{C} 11-\mathrm{H} 11 \mathrm{~A}$ & 0.9700 \\
\hline $\mathrm{C} 6-\mathrm{N} 2$ & $1.342(3)$ & C11-H11B & 0.9700 \\
\hline $\mathrm{C} 6-\mathrm{N} 1$ & $1.383(3)$ & $\mathrm{C} 12-\mathrm{H} 12 \mathrm{~A}$ & 0.9700 \\
\hline $\mathrm{C} 7-\mathrm{C} 12$ & $1.512(4)$ & $\mathrm{C} 12-\mathrm{H} 12 \mathrm{~B}$ & 0.9700 \\
\hline $\mathrm{C} 7-\mathrm{C} 8$ & $1.530(3)$ & & \\
\hline $\mathrm{N} 1-\mathrm{C} 1-\mathrm{C} 7$ & $123.0(2)$ & $\mathrm{H} 8 \mathrm{~A}-\mathrm{C} 8-\mathrm{H} 8 \mathrm{~B}$ & 108.0 \\
\hline $\mathrm{N} 1-\mathrm{C} 1-\mathrm{S} 1$ & $115.61(17)$ & $\mathrm{C} 10-\mathrm{C} 9-\mathrm{C} 8$ & $111.3(2)$ \\
\hline $\mathrm{C} 7-\mathrm{C} 1-\mathrm{S} 1$ & $121.33(17)$ & $\mathrm{C} 10-\mathrm{C} 9-\mathrm{H} 9 \mathrm{~A}$ & 109.4 \\
\hline $\mathrm{C} 3-\mathrm{C} 2-\mathrm{C} 6$ & $119.8(2)$ & $\mathrm{C} 8-\mathrm{C} 9-\mathrm{H} 9 \mathrm{~A}$ & 109.4 \\
\hline $\mathrm{C} 3-\mathrm{C} 2-\mathrm{S} 1$ & $131.07(19)$ & $\mathrm{C} 10-\mathrm{C} 9-\mathrm{H} 9 \mathrm{~B}$ & 109.4 \\
\hline $\mathrm{C} 6-\mathrm{C} 2-\mathrm{S} 1$ & $109.08(17)$ & $\mathrm{C} 8-\mathrm{C} 9-\mathrm{H} 9 \mathrm{~B}$ & 109.4 \\
\hline $\mathrm{C} 4-\mathrm{C} 3-\mathrm{C} 2$ & $116.4(2)$ & $\mathrm{H} 9 \mathrm{~A}-\mathrm{C} 9-\mathrm{H} 9 \mathrm{~B}$ & 108.0 \\
\hline $\mathrm{C} 4-\mathrm{C} 3-\mathrm{H} 3$ & 121.8 & $\mathrm{C} 9-\mathrm{C} 10-\mathrm{C} 11$ & $111.2(2)$ \\
\hline $\mathrm{C} 2-\mathrm{C} 3-\mathrm{H} 3$ & 121.8 & $\mathrm{C} 9-\mathrm{C} 10-\mathrm{H} 10 \mathrm{~A}$ & 109.4 \\
\hline $\mathrm{C} 3-\mathrm{C} 4-\mathrm{C} 5$ & $120.2(2)$ & $\mathrm{C} 11-\mathrm{C} 10-\mathrm{H} 10 \mathrm{~A}$ & 109.4 \\
\hline $\mathrm{C} 3-\mathrm{C} 4-\mathrm{H} 4$ & 119.9 & $\mathrm{C} 9-\mathrm{C} 10-\mathrm{H} 10 \mathrm{~B}$ & 109.4 \\
\hline $\mathrm{C} 5-\mathrm{C} 4-\mathrm{H} 4$ & 119.9 & $\mathrm{C} 11-\mathrm{C} 10-\mathrm{H} 10 \mathrm{~B}$ & 109.4 \\
\hline $\mathrm{N} 2-\mathrm{C} 5-\mathrm{C} 4$ & $124.9(2)$ & $\mathrm{H} 10 \mathrm{~A}-\mathrm{C} 10-\mathrm{H} 10 \mathrm{~B}$ & 108.0 \\
\hline $\mathrm{N} 2-\mathrm{C} 5-\mathrm{H} 5$ & 117.6 & $\mathrm{C} 10-\mathrm{C} 11-\mathrm{C} 12$ & $111.0(3)$ \\
\hline $\mathrm{C} 4-\mathrm{C} 5-\mathrm{H} 5$ & 117.6 & $\mathrm{C} 10-\mathrm{C} 11-\mathrm{H} 11 \mathrm{~A}$ & 109.4 \\
\hline
\end{tabular}




$\begin{array}{llll}\mathrm{N} 2-\mathrm{C} 6-\mathrm{N} 1 & 121.2(2) & \mathrm{C} 12-\mathrm{C} 11-\mathrm{H} 11 \mathrm{~A} & 109.4 \\ \mathrm{~N} 2-\mathrm{C} 6-\mathrm{C} 2 & 123.3(2) & \mathrm{C} 10-\mathrm{C} 11-\mathrm{H} 11 \mathrm{~B} & 109.4 \\ \mathrm{~N} 1-\mathrm{C} 6-\mathrm{C} 2 & 115.5(2) & \mathrm{C} 12-\mathrm{C} 11-\mathrm{H} 11 \mathrm{~B} & 109.4 \\ \mathrm{C} 1-\mathrm{C} 7-\mathrm{C} 12 & 113.3(2) & \mathrm{H} 11 \mathrm{~A}-\mathrm{C} 11-\mathrm{H} 11 \mathrm{~B} & 108.0 \\ \mathrm{C} 1-\mathrm{C} 7-\mathrm{C} 8 & 109.77(19) & \mathrm{C} 7-\mathrm{C} 12-\mathrm{C} 11 & 111.5(3) \\ \mathrm{C} 12-\mathrm{C} 7-\mathrm{C} 8 & 110.3(2) & \mathrm{C} 7-\mathrm{C} 12-\mathrm{H} 12 \mathrm{~A} & 109.3 \\ \mathrm{C} 1-\mathrm{C} 7-\mathrm{H} 7 & 107.8 & \mathrm{C} 11-\mathrm{C} 12-\mathrm{H} 12 \mathrm{~A} & 109.3 \\ \mathrm{C} 12-\mathrm{C} 7-\mathrm{H} 7 & 107.8 & \mathrm{C} 7-\mathrm{C} 12-\mathrm{H} 12 \mathrm{~B} & 109.3 \\ \mathrm{C} 8-\mathrm{C} 7-\mathrm{H} 7 & 107.8 & \mathrm{C} 11-\mathrm{C} 12-\mathrm{H} 12 \mathrm{~B} & 109.3 \\ \mathrm{C} 9-\mathrm{C} 8-\mathrm{C} 7 & 111.1(2) & \mathrm{H} 12 \mathrm{C} 12-\mathrm{H} 12 \mathrm{~B} & 108.0 \\ \mathrm{C} 9-\mathrm{C} 8-\mathrm{H} 8 \mathrm{~A} & 109.4 & \mathrm{C} 1-\mathrm{N} 1-\mathrm{C} 6 & 110.6(2) \\ \mathrm{C} 7-\mathrm{C} 8-\mathrm{H} 8 \mathrm{~A} & 109.4 & \mathrm{C} 5-\mathrm{N} 2-\mathrm{C} 6 & 115.3(2) \\ \mathrm{C} 9-\mathrm{C} 8-\mathrm{H} 8 \mathrm{~B} & 109.4 & \mathrm{C} 2-\mathrm{S} 1-\mathrm{C} 1 & 89.19(10) \\ \mathrm{C} 7-\mathrm{C} 8-\mathrm{H} 8 \mathrm{~B} & 109.4 & & \end{array}$

